\title{
EL INFINITO DESDE LA FÍSICA
}

\author{
ÁLVARO BALSAS, SJ \\ Universidade Católica Portuguesa, Braga
}

RESUMEN: En física, el infinito aparece vinculado al problema de lo continuo de las representaciones matemáticas usadas por las diversas teorías y, por lo tanto, a la posibilidad de la infinita divisibilidad (lo infinitamente pequeño) y de la infinita extensión (lo infinitamente grande) de la geometría del espaciotiempo y de los conceptos y magnitudes físicas mensurables. El modelo estándar de las partículas considera que las partículas elementales son «infinitamente» pequeñas, donde surgen cálculos con resultados infinitos para determinadas cantidades físicas y, para eliminarlos, se utilizan métodos de renormalización, usados también en la cosmología clásica y en el modelo estándar cosmológico del Big Bang con la finalidad de domesticar los infinitos (agujeros negros y singularidad inicial). La investigación en gravedad cuántica considera que el espacio no sea infinitamente divisible y los modelos de multiversos suponen tiempos y espacios infinitos para el Universo. Siendo nuestras teorías siempre limitadas y perfectibles, parece que no podemos probar, en física, que el Universo sea físicamente infinito.

PALABRAS CLAVE: infinito; física de partículas; cosmología del big bang; gravedad cuántica; multiversos.

\section{Infinity from Physics}

ABSTRACT: In physics, infinity appears linked to the continuum problem of the mathematical representations used by the various theories and, therefore, to the possibility of infinite divisibility (the infinitely small) and the infinite extension (the infinitely large) of the geometry of space-time and of the measurable physical concepts and magnitudes. The standard model of particle physics considers that elementary particles are «infinitely» small and calculations arise with infinite results for certain physical quantities. To eliminate those infinities, we use methods of renormalization, which are also used in classical cosmology and in the standard model of the Big Bang cosmology in order to tame the infinities (black holes and initial singularity). Research in quantum gravity considers that space is not infinitely divisible and multiverse models assume infinite times and spaces for the Universe. Since our theories are always limited and perfectible, it seems that we cannot prove, in physics, that the Universe is physically infinite.

KEY WORDS: infinity; particle physics; big bang cosmology; quantum gravity; multiverses.

\section{INTRODUCCIÓN}

Afirma Aristóteles en su III libro de la Física que,

«Dado que la Ciencia de la Naturaleza se ocupa de magnitudes, movimiento y tiempo, cada uno de los cuales tiene que ser finito o infinito (...), sería conveniente que quien estudia la Naturaleza considere, sobre lo infinito, si existe o no; y si existe, qué cosas es».

Añade aún el estagirita que todos los antiguos filósofos de la Naturaleza (es decir, los que, entonces, eran llamados físicos, o estudiosos de la physis): 
«[H]an dado una explicación sobre lo infinito. Todos también lo establecen como un principio (arché) de las cosas-que-son (...). Platón, por su parte, sostiene que las cosas infinitas son dos: lo grande y lo pequeño» ${ }^{1}$.

Ya al comienzo de ese mismo libro, Aristóteles había afirmado que:

«Parece que el movimiento pertenece a las cosas continuas y lo infinito es lo primero que se manifiesta en lo continuo; razón por la que acaece también que quienes tratan de definir lo continuo utilizan a menudo adicionalmente la noción de infinito, en la idea de que es continuo lo que es divisible hasta el infinito. Pero además de esto es imposible que exista el movimiento al margen del lugar, el vacío y el tiempo»².

Un poco más adelante el estagirita considera, con base en su teoría hilemórfica de descripción de las cosas en términos del acto y de la potencia, que el infinito no puede existir en el mismo modo que un cuerpo finito, pues, para él:

«Aunque lo más apropiado para el Filósofo de la Naturaleza es considerar si existe una magnitud perceptible infinita. (...) lo infinito es, en suma, o en virtud de adición o en virtud de división o de ambas maneras. Pues bien, que lo infinito sea separable de las cosas perceptibles, siendo algo infinito en sí, no es posible. (...) Y está claro también que no es posible que lo infinito exista como "siendo en actualidad" (energeía) y también como entidad (ousía, sustancia) y principio (arché). (...) Así pues, de esto resulta claro que en actualidad no existe cuerpo infinito. Nos resta, pues, que lo infinito sea en potencia $»^{3}$.

O sea, para este filósofo lo infinito no puede existir como infinito actual (como una acción terminada), sino, más bien, como infinito potencial, en potencia, porque es la posibilidad de una acción sin término de un proceso inacabado o incompleto, obtenido por adición o división de partes sucesivas:

«El infinito es así por el hecho de tomarse sucesivamente una y otra vez, y por el hecho de que lo que se toma siempre es finito, aunque siempre, desde luego, sucesivamente diferente» ${ }^{4}$.

En resumen, para Aristóteles el infinito no existe como una entidad actualizada, sino solamente como una potencialidad de adición o de división ilimitada, como, por ejemplo, en una recta infinita de puntos, donde, entre dos puntos cualesquiera, por más próximos que estén entre sí, siempre cabe «uno punto más»; o en la división de un segmento de recta en trozos sucesivamente menores. El infinito es, pues, un modo de potencialidad asociado a un proceso de adición o división sucesivas (es infinito matemático), pero nunca como completamente actualizado como infinito sustancial (sin realización efectiva de la serie total como un todo

1 Aristóteles, Física, III, 4, 203a, pp. 71, 72 (citamos en este trabajo las páginas del texto revisado y traducido por José Luis Calvo Martínez de la edición del Consejo Superior de Investigaciones Científicas, Madrid, 1996).

$2 \quad$ Ibid., Física, III, 1, 200b, pp. 63.

$3 \quad$ Ibid., Física, III, 4-5, 204a, pp. 74-75; III, 5-6, 206a, p. 81.

$4 \quad$ Ibid., Física, III, 6, 206a, p. 82. 
o totalidad). Por otras palabras, el infinito de Aristóteles es un infinito potencial. En realidad, en el proceso de adición sucesiva, la magnitud adicionada no tiene limitación (o cota) superior, por lo que hablamos en infinitamente grande; en el proceso de división sucesiva la magnitud dividida no tiene límite inferior, salvo el cero. Los matemáticos definen los infinitos, tanto grandes como pequeños, merced de un proceso de paso al límite.

Evidentemente que la física moderna, que tiene sus comienzos en el siglo XVII, con Galileo (1564-1642), está muy distante, en sus métodos y en sus teorías, de la física aristotélica. De hecho, la física de Galileo ya no busca descubrir las esencias de las entidades naturales y sus relaciones jerárquicas, sino investigar las leyes que rigen los fenómenos naturales, basando-se en la unión de la experimentación con la matemática, ya que, de acuerdo con el mismo Galileo, «el libro de la naturaleza está escrito en lenguaje matemático». Por ello, interesan ahora, en la revolución científica del siglo XVII, las calidades físicas, objetivas (o primarias) de los fenómenos naturales, que son cuantificables y mensurables, es decir, aquellas capaces de ser medidas a través de instrumentos de los cuales emergen números y que pueden ser expresadas por relaciones matemáticas. Este nuevo método científico de abordar la Naturaleza vale sea para las teorías de la física clásica de Newton, Maxwell o de Einstein, sea para la física cuántica.

Todavía, el problema del infinito, cuando se coloca en las diferentes teorías de la física desde Galileo hasta el presente, surge de cuestiones similares a aquellas que han sido formuladas por Aristóteles. Es decir, el infinito surge vinculado a las cuestiones sobre la continuidad, divisibilidad y extensión del espacio, del tiempo, y de las magnitudes de las propiedades físicas de la materia. Avancemos desde ya que en física el concepto o noción de infinito no es un buen concepto físico; en principio ninguna idea física debería apelar a nociones infinitas, pues no medimos nunca en nuestros instrumentos cantidades infinitas. Así, en física, el infinito surge no como un "hecho» físico, resultante de una medición experimental, sino como un límite matemático de la teoría, es decir, como una demostración de que la teoría física ha fallado, por lo menos parcialmente, en la descripción de los fenómenos que pretende describir. Para mejor entender el significado de esta última afirmación necesitamos de ver más en detalle en qué consiste una teoría física.

\section{1. ¿QUÉ ES UNA TEORÍA FÍSICA? La ARQUITECTURA DE LA FÍSICA}

La física ofrece un conjunto de representaciones teóricas para describir y explicar los fenómenos naturales, pasibles de ser observados y medidos, que percibimos en el Mundo. La observación capta, a través de mediciones, una alteración de un sistema natural, que sufre un cambio finito, y por ello mensurable, de un estado inicial para un estado final. En esa alteración hay, por lo tanto, «entidades» o «objetos» que cambian, en el espacio y en el tiempo. Para describir nuestra observación y experiencia del Mundo usamos un lenguaje con determinados concep- 
tos que puedan de alguna manera articular nuestras percepciones del Mundo y la estructura del mismo Mundo, de tal modo que la estructura del lenguaje usado y la estructura del Mundo se condicionan mutuamente ${ }^{5}$.

El lenguaje por antonomasia de la física es el lenguaje de las matemáticas, que en los últimos siglos ha conocido estruendosos desarrollos. La singular adecuación de la matemática en la representación de la Naturaleza ha intrigado desde siempre a filósofos, físicos e matemáticos. La precisión del lenguaje matemático junto con su estructura lógica formal hace que sus proposiciones puedan tener sólo un significado bien determinado, unívoco e universal, dotando así a la física con un enorme carácter predictivo de revelarnos el Cosmos. Por ejemplo, cuando Maxwell combinó los campos eléctrico y magnético en uno solo campo electromagnético, el formalismo de sus ecuaciones diferenciales reveló paralelamente que la luz era un fenómeno electromagnético. Un segundo ejemplo, la ecuación relativista de Dirac de la Mecánica Cuántica reveló que a cada partícula material corresponde una partícula de anti-materia que le está asociada.

La física describe la evolución dinámica del Cosmos a partir de dos categorías conceptuales indisociables: «los objetos-actores y el espacio-tiempo-arena ${ }^{6}$. Las teorías o modelos físicos son construcciones abstractas de nuestro intelecto, que pretenden representar, describir y explicar el Cosmos. Esas teorías asientan en una base axiomática (que llamamos postulados o principios), a partir de los cuales se deducen lógicamente, con el auxilio de conceptos (por ejemplo, tiempo, espacio, masa, carga eléctrica, energía, espín, etc.), sus leyes (que enlazan los diversos conceptos entre sí), resultados, conclusiones y previsiones, los cuales podrán ser contrastados con la observación empírica.

Los principios o postulados no son demostrables dentro de la teoría y deben ser suficientemente generales (para abarcar el mayor número posible de sistemas naturales) y en menor número posible. Por ejemplo, en la Teoría General de la Relatividad (TGR) hay solamente dos principios básicos: el Principio de la Relatividad — que afirma que todos los sistemas de observación son igualmente equivalentes, o sea, que las leyes de la física tienen la misma forma en todos los sistemas de referencia- y el Principio de Equivalencia -que establece la equivalencia entre gravitación y aceleración (o entre las masas gravitacional e inercial) ${ }^{7}$.

Los conceptos son introducidos mediante definiciones arbitrarias, pero que se desean que representen cualidades físicas de los sistemas naturales y que, por ello, pueden ser medidas. Por ejemplo, Newton empieza su libro de los Principia (Philosophiae Naturalis Principia Mathematica) con ocho definiciones (la cantidad de materia, la cantidad de movimiento, la fuerza ínsita o inercia

\footnotetext{
5 Videira, A. L. L., «O Mundo que nos pertence», Ciência \& Ambiente, v. 28, Janeiro / Junho 2004, pp. 140-143.

6 Videira, A. L. L., «O Mundo que nos pertence», Ciência \& Ambiente, v. 28, Janeiro / Junho 2004, p. 143.

7 Videira, A. L. L., "A(s) Relatividade(s) de Einstein», Ciência \& Ambiente, v. 30, Janeiro / Junho 2005, pp. 72-76.
} 
de la materia, la fuerza ejercida sobre un cuerpo, la fuerza centrípeta, la magnitud absoluta de la fuerza centrípeta, la su magnitud aceleradora y su magnitud motriz), siguiéndose los 3 axiomas o leyes del movimiento y sus corolarios.

En física, existen también los llamados modelos, que se distinguen de las teorías por presentar menor nivel de exigencia formal que estas últimas, aunque manteniendo el mismo nivel de exigencia de validación empírica. Así, los modelos son construcciones menos conseguidas que las teorías, sea por su elaboración formal, menos exigente, o por contener deficiencias en su estructura, sea por su capacidad predictiva, más limitada. Por ejemplo, hablamos de Teoría de Maxwell del electromagnetismo, de las dos teorías de la Relatividad de Einstein y de Teoría de la Mecánica Cuántica (MC); pero hablamos del Modelo de Bohr para el átomo, anterior a la formulación de la MC, o del Modelo Estándar de la Física de Partículas. Ambos son considerados construcciones transitorias, mientras no dispongamos de teorías comprensibles deducida de postulados.

Subrayemos que el contraste que se hace entre teoría o modelo y el mundo de los fenómenos físicos es, en realidad, un contraste entre los números que salen de esa teoría o modelo y los números que salen de los experimentos cuantificables. Recordemos también que los números resultantes de experimentos científicos nunca son infinitos, dado que el experimento es siempre una interacción, de duración finita, entre un aparato de medición y el sistema medido, produciendo un cambio, siempre finito, en alguna propiedad física de ese sistema.

\section{LO INFINITO EN LA FísICA ACTUAL}

Las grandes teorías de la física actual son la Teoría Especial de la Relatividad (TER, de 1905), la Teoría General de la Relatividad (TGR, 1915) de Einstein y la Mecánica Cuántica (MC, 1925). La primera teoría de la relatividad nos proporciona una formulación del espacio-tiempo y de la mecánica compatible con la teoría de Maxwell; la segunda nos proporciona una formulación de la gravedad y una nueva concepción de la geometría espacio-temporal que, en particular permite una nueva representación de la evolución del Universo. La MC nos proporciona una formulación de los fenómenos atómicos y subatómicos. Su geometría espaciotemporal es aún considerada como continua (en la que se puede utilizar el cálculo diferencial e integral), mientras que las cantidades mensurables características de los objetos cuánticos (como, por ejemplo, la masa y el espín) son consideradas como discretas.

La TGR sustituye la teoría gravitacional de Newton del espacio y del tiempo, que consideraba el espacio como un substractum continuum sin propiedades dinámicas y el tiempo como una propiedad igualmente continua, siendo que los cuerpos, debido a las fuerzas mecánicas de interacción mutua, evolucionarían 
dinámicamente de un modo continuo en el espacio y en el tiempo (ambos considerados teóricamente como continuos e infinitos), de acuerdo con la ecuación de Newton, que es una ecuación diferencial. O sea, la representación formal de la física newtoniana exige una fondo, o geometría, que consiste en el producto de cuatro rectas infinitas, mutuamente ortogonales, una para el tiempo y tres para el espacio. Recordemos que la física de Newton, que sigue el Principio de Relatividad de Galileo y las transformaciones de Galileo, como su base axiomática, admite la posibilidad de sistemas físicos que se dislocan a velocidades arbitrariamente elevadas (e, por lo tanto, teóricamente infinitas), siendo esto compatible con el concepto de simultaneidad absoluta de los acontecimientos. En el espacio-tiempo de Newton, con geometría plana, actúa una misteriosa fuerza de gravedad (que se propaga instantáneamente a distancia, o sea, con una velocidad infinita). Así, en la concepción de Newton para la representación de la gravitación existe el fondo del espacio-tiempo y la acción de la gravitación, que es una fuerza.

En la TGR el espacio-tiempo es interpretado como un fondo continuo con propiedades dinámicas co-determinadas por la materia-energía de los objetos en acción. Es decir, el espacio newtoniano es ahora «reinterpretado como una sustancia continua que puede estirarse y doblarse como una lámina de goma, (...) de acuerdo con las ecuaciones de Einstein ${ }^{8}$. En otras palabras, el espaciotiempo está ahora dotado de curvatura, prescindiendo de la intervención de cualquier fuerza para reproducir el fenómeno gravitacional. Ahora, la gravitación es representada como efecto de la geometría espacio-temporal sobre el movimiento de los sistemas físicos - la materia y la radiación - en ese fondo cuadridimensional, de tal modo que el espacio-tiempo actúa sobre la materia y la radiación, dictándoles cómo se deben dislocar. A su vez, la materia y la radiación actúan sobre el espacio-tiempo, dictándole como se debe curvar. Así explica Einstein la gravitación. Se puede visualizar esta concepción de la gravitación a través de una membrana elástica que se deforma por la presencia de un objeto con determinada masa.

Subrayemos de nuevo que hay una diferencia muy significativa entre las teorías de Newton y de Einstein. La primera supone, desde el inicio, la existencia de un fondo pre-fijado del espacio y del tiempo absolutos, donde van ocurrir los procesos físicos materiales. La segunda no supone ese fondo previo, sino que ese fondo es determinado por (o nace de) las entidades dinámicas; y el dinamismo de estas es determinado por la geometría del fondo. O sea, la TGR une dos caras o faces: una geométrica (la arena del espacio-tiempo) y otra, la de las entidades dinámicas (los actores materia y radiación), que se co-determinan mutuamente, pues están las dos explícitamente entrelazadas en las ecuaciones fundamentales del campo gravitacional, las ecuaciones de

Rovelli, Carlo, M., "Some Considerations on Infinity in Physics», en: Heller, M. \& Woodin, H. (eds.), Infinity: New Research Frontiers, Cambridge University Press, Cambridge, 2011, p. 168. 
Einstein, que son ecuaciones no lineales de segundo orden, cuyo contenido traduce, grosso modo, que ${ }^{9}$ :

\section{\{La curvatura del espacio-tiempo $3+1$ dimensional $\}=$ $=\{$ Densidad de materia y radiación $\}$.}

En resumen, la teoría de Newton une el espacio-tiempo newtoniano con las trasformaciones de Galileo. Para esta teoría, espacio y tiempo son cantidades continuas que teóricamente pueden tomar valores de cero a infinito; no tienen, pues, límites. La teoría de la Relatividad Especial junta el espacio-tiempo de Minkowski con las trasformaciones lineales de Lorentz. Pero, en esta teoría hay un límite superior finito para la propagación de los fenómenos físicos (y, por lo tanto, para la información), que es la velocidad de la luz en el vacío. Todavía, el tiempo puede tender al infinito, debido a la dilatación temporal (o sea, el tiempo se va parando), mientras que el espacio correspondiente, por estar intrínsecamente entrelazado con el tiempo, se va contrayendo o reduciendo, teóricamente hasta cero. Finalmente, la TGR une un espacio-tiempo de Riemann con las trasformaciones lineales generales, manteniendo la finitud de la velocidad de propagación de los fenómenos físicos, limitada por la velocidad de luz en el vacío. Además de todos los efectos gravitatorios habituales, la TGR describe de manera satisfactoria la evolución del Universo a gran escala. En esta teoría aparecen infinitos, llamados singularidades, que son los agujeros negros y el Big Bang (BB) o comienzo del Universo.

Conviene decir que la TGR de Einstein tiene sido sometida a pruebas experimentales cada vez más exigentes, siendo los resultados consistentes con la teoría. Las últimas los últimas pruebas se refieren a las ondas gravitacionales, que transportan energía gravitacional, debida a oscilaciones del espaciotiempo, originadas por masas en movimiento acelerado. Estas ondas han sido detectadas, el 14 de septiembre de 2015, por el observatorio norteamericano LIGO, compuesto por 2 interferómetros separados por una distancia de 3 mil km (Livingston, Luisiana y Hanford, Washington). La señal detectada ha sido originada por una violentísima colisión de dos agujeros negros en interacción gravitacional (con masas iguales a 36 y 29 veces la masa del Sol), en el centro de una galaxia situada a una distancia de mil y trecientos millones de años-luz. Este resultado es la primera evidencia directa no sólo de ondas gravitacionales, sino también de agujeros negros.

En MC las propiedades dinámicas mensurables de los objetos no pueden admitir valores continuos, sino que son descontinuas o cuantizadas, es decir, cuando estos objetos son observados manifiestan sus propiedades en cuantidades discretas, múltiplos de unos valores mínimos, designados por «cuanta». Por ejemplo, ondas electromagnéticas observadas en escala de

9 Videira, A. L. L., «"Um dos Maiores Se Não Mesmo o Maior dos Feitos na História do Pensamento Humano": A Teoria da Relatividade Geral», Monografia, Centro Brasileiro de Pesquisas Físicas, CBPF, v. 2, n. 1, 2016, p. 5. 
intensidad suficientemente pequeña ${ }^{10}$ muestran comportarse como partículas individuales, los fotones, cuyas propiedades son múltiplos de ciertos valores mínimos posibles. La MC fue seguida de su extensión relativista y a mediados del siglo XX se ha desarrollado la Teoría Cuántica de Campos (la llamada segunda cuantización) para unir los conceptos de la MC con los de la Relatividad.

En la primera cuantización los objetos fundamentales que intervienen en los fenómenos físicos, como componentes básicas del Cosmos, son las llamadas partículas elementales (y sus anti-partículas) y los campos. Las primeras son fuentes de estos últimos, sirviendo estos para aquellas interaccionaren. En la segunda cuantización, los elementos básicos son los campos, siendo las partículas consideradas meramente como los cuanta de los respectivos campos (por ejemplo, los fotones son los cuanta del campo electromagnético).

Las dos teorías describen las cuatro interacciones actualmente conocidas. La MC es responsable de las interacciones electromagnética, nuclear fuerte y nuclear débil, y la TGR describe la interacción gravitacional. Contrariamente al caso de los fenómenos electromagnéticos y nucleares, que ya disponen de una descripción relativista cuántica, el fenómeno de la gravitación — si no se acepta la teoría de las cuerdas como una descripción satisfactoria- continúa hasta hoy resistiendo firmemente a todos los esfuerzos en el sentido de construir una Teoría Cuántica de la Gravitación, pues los supuestos de las dos teorías son muy diferentes. En realidad, la TGR ha alterado, con relación a Newton, el concepto de espacio-tiempo, manteniendo intacta la relación entre observador y observado, siendo la interacción entre ambos descrita en términos continuos. A su vez, la Mecánica Cuántica, ha alterado la relación entre observador y observado, describiendo la interacción entre ambos en términos descontinuos, pero manteniendo la concepción newtoniana del espacio-tiempo. Así, se hacen comprensibles las dificultades de la física actual en unificar las dos teorías, con supuestos diferentes, y en llegar a una única teoría - la Teoría Cuántica de Gravitación - que pueda describir de modo unificado y coherente las cuatro interacciones conocidas para los fenómenos físicos, referidas anteriormente.

En los intentos actuales por unificar las dos teorías - desarrollados, por ejemplo, por la teoría de cuerdas o por la «gravedad cuántica de lazos» (o de loops)—, al abordar cuestiones relativas a la estructura microscópica del espacio-tiempo y al origen, evolución y estructura del Universo como un todo, surgen singularidades y divergencias (valores infinitos para las variables) en las representaciones cuánticas del Cosmos, lo que exige re-normalizaciones ad hoc para transformar las cantidades infinitas en finitas, ya que en física no medimos cantidades infinitas. En los últimos cuarenta años se ha trabajado en la Teoría de las Cuerdas, como teoría fundamental de las partículas elementales, que busca lograr una descripción unificada de todas las interacciones fundamentales de la naturaleza, incluyendo la cuantización de la gravitación. Pero, esta no se ha afirmado convincentemente sea a nivel formal (hay varias teorías diferentes,

10 Hoy ya se consiguen experiencias envolviendo la emisión de un único fotón a la vez. 
que postulan números diferentes de dimensiones para el Universo, pero siempre superior a cuatro), sea a nivel de verificación experimental (que exige condiciones muy difíciles de alcanzar actualmente).

\section{Lo INFINITAMENTE PEQUEÑO Y LA FÍSICA DE LAS PARTícUlaS}

Lo infinitamente pequeño en física es abordado por la física de partículas. El modelo estándar de la física de partículas (para señalar que se trata de una teoría con un rigor formal todavía insuficiente o incompleto, en proceso de elaboración) describe la dinámica de todas las partículas elementales conocidas a partir de tres de las cuatro interacciones fundamentales también conocidas (electromagnética, fuerte y débil), pues la gravitación, por el momento, ha sido dejada aparte. Las interacciones electromagnética y gravitacional son de largo alcance, teóricamente de alcance infinito ${ }^{11}$; las otras dos son de corto alcance $^{12}$. Sin embargo, este modelo tiene dos limitaciones básicas (además de otras limitaciones): no explica por qué la naturaleza es descrita por este conjunto de partículas e interacciones y, por otra parte, no da cuenta de la cuarta interacción fundamental que es la gravitación (ni de la interacción que rige la energía oscura).

La materia normal o bariónica es constituida por 6 quarks $(u, d, c, s, t, b$; arriba, abajo, encanto, extraño, cima, fondo) y 6 leptones (electrón, muon, tau, y los neutrinos, electrónico, muónico y tauónico), pudiendo ser agrupada en tres familias leptón-quark (dos de cada). Los quarks son los componentes de protones y neutrones, experimentando las tres interacciones, pero los leptones son inmunes a la interacción fuerte. Lo que diferencia a los dos tipos de partículas es el «color» (hay tres tipos de color), una propiedad de los quarks (que sufren la interacción fuerte, transmitida por ocho gluones «coloreados»), que guarda semejanza lejana con la carga eléctrica. Así, los quarks poseen color y los leptones no. El modelo estándar considera que las interacciones son trasmitidas por las partículas portadoras o mediadoras. Así, tenemos los mediadores de la interacción electromagnética —el fotón-, de la interacción fuerte - los ocho gluones «coloredos»-, de la interacción débil —los bosones $\mathrm{W}^{+}, \mathrm{W}^{-}$y $\mathrm{Z}^{0}$ - , y el bosón de Higgs $\mathrm{H}$, responsable por la atribución de masa a las partículas elementales.

La cosmología actual nos dice que la materia bariónica (de la que están hechas las estrellas, los planetas y nosotros), visible electromagnéticamente, representa solo el 5\% (cinco por ciento) de la materia-energía del Universo. Hay evidencias de que, asociada a la componente visible de cada galaxia, existe una distribución invisible de materia (un halo invisible, por veces más extenso

11 Como el alcance de las dos interacciones varía con la inversa del cuadrado de la distancia entre la fuente del campo y el observador, tal alcance sólo se anula en el infinito.

12 Su alcance se anula para distancias superiores al diámetro de un núcleo atómico. 
que la galaxia) diversa de la materia normal: es la materia oscura, que se estima que sea un $27 \%$, o sea, aproximadamente un cuarto del total. Además, desde 1998, se habla también de la energía oscura, responsable de la aceleración de la expansión cósmica (funcionando como una anti-gravedad), y que corresponde a $68 \%$ de la materia-energía total del Universo.

Conviene subrayar que en el marco de las teorías cuánticas de campos - la electrodinámica cuántica, que describe la cuantización de la interacción electromagnética, mediada por fotones, y la cromodinamica cuántica, que describe la interacción fuerte, que se da entre quarks y es vehiculada por gluones-, usadas por el modelo estándar de las partículas, se considera que todas las partículas elementales son «infinitamente» pequeñas, es decir, no tienen estructura interna, ni dimensión mensurable hasta hora. En otras palabras, esas partículas son consideradas «locales», o sea, están confinadas en un volumen infinitamente pequeño o un punto sin dimensiones. Puesto que la teoría de campos está fundada sobre la noción de interacciones locales, lo infinitamente pequeño es omnipresente en ella. Por ejemplo, en la EDQ un electrón teórico es una partícula puntual, aunque dotada de masa, carga eléctrica y otras propiedades, siendo capaz de participar en interacciones electromagnéticas. Pero, en los experimentos, no hay resultados infinitos. A consecuencia del principio de incertidumbre de Heisenberg, se considera que el electrón no está completamente desnudo, sino que está rodeado de una «nube de partículas virtuales» debido a la polarización del vacío cuántico (donde hay permanente creación de pares virtuales electrón-positrón) ${ }^{13}$. Cuando, en finales de los años 1940 se calculó la carga y la masa del electrón desnudo, tomando en consideración los efectos de la polarización del vacío, los cálculos llevaban a resultados infinitos, lo que era inaceptable. Tal absurdo era consecuencia de la hipótesis de electrones desnudos puntuales y en interacción local. Para eliminar de los cálculos los resultados infinitos, se usó el método de la renormalización, en el que, prescindiendo de la carga del electrón desnudo, se ajustan la carga y la masa del electrón «vestido» a los resultados experimentales. Así las cantidades infinitas quedan eliminadas y los resultados obtenidos para las magnitudes físicas medibles son de una gran precisión. El problema de los infinitos en las teorías locales, que hacen intervenir partículas puntuales se ha propuesto también para la cromodinámica cuántica.

¿Qué ocurre ahora cuando, a distancias muy pequeñas, consideramos también la gravedad? La conjunción de, por una parte, del modelo estándar, con sus teorías cuánticas (conteniendo discontinuidades) y sus interacciones locales entre partículas puntuales, y, por otra, de la relatividad general, una

13 La física cuántica asegura que en el vacío hay formación y destrucción continua de partículas virtuales (fluctuaciones del vacío), que se crean en pares partícula-antipartícula que se aniquilan y desaparecen en intervalos de tiempo ínfimos. Estas partículas pueden interaccionar entre sí y se han observado efectos experimentales de este hecho, de tal modo que se considera la energía del vacío tan real como las propias partículas elementales de la materia bariónica. 
teoría clásica que considera que la gravitación es un efecto de la deformación (continua) del espacio-tiempo (que también presenta singularidades), exigiría cuantizar al espacio-tiempo, ya que a distancias muy pequeñas (inferiores a 1035 metros) las incertidumbres cuánticas perturban probablemente la estructura del espacio-tiempo. Así, por ejemplo, a esta escala pequeña, las singularidades espacio-temporales de un electrón y de todas las otras partículas elementales tendrían que desaparecer. Esta es la idea básica de las teorías de supercuerdas. Estas teorías postulan que los auténticos componentes fundamentales de la materia para energías muy altas (del orden de la energía de Planck, $10^{19} \mathrm{GeV}$ ) no son objetos puntuales, sino objetos extensos con estructura (semejantes a membranas de dimensiones muy pequeñas), llamados cuerdas (que pueden ser abiertas o cerradas sobre sí mismas, como unos tubos).

Mientras una partícula que se mueve en el espacio describe una línea, una cuerda barre una superficie al desplazarse en el espacio. Si la masa es una de las magnitudes características de las partículas, la tensión de la cuerda es la magnitud característica de la inercia de la cuerda. Las diferentes partículas elementales corresponden a diferentes vibraciones de las cuerdas. En cuerdas con una tensión muy grande, los puntos apenas se pueden separar y, de este modo, se recupera la noción de partícula elemental. Así, solo a energías muy grandes, la estructura no puntual de las cuerdas se hace manifiesta.

Con estas teorías de cuerdas, que son actualmente de varios tipos, se intenta solucionar el problema de las cantidades infinitas que aparecen en los cálculos de una teoría de la gravitación cuántica. Pero, en estas teorías, el espacio-tiempo es ahora más insólito, pues requiere nueve (o diez) dimensiones espaciales (o sea, 5 o 6 dimensiones suplementarias adicionales a las dimensiones habituales: arriba-abajo, derecha-izquierda, delante-detrás) y una dimensión temporal. De estas diez u once dimensiones, sólo las cuatro dimensiones observables tienen dimensiones macroscópicas.

Podemos utilizar una analogía para ilustrar lo que acabamos de decir. Si enrollamos una hoja de papel, dándole forma cilíndrica con un radio pequeño, y la vemos desde lejos, ella se asemejará a un segmento de recta, con una sola dimensión. Pero, hay una segunda dimensión, que es la que permite dar la vuelta en torno del cilindro, la cual no se percibe sino a corta distancia de la hoja. Así ocurriría con las seis (o siete) dimensiones suplementarias, encorvadas, de las teorías de cuerdas, que tendrían una importancia fundamental en pequeñas escalas y que se encontrarían confinadas en dominios ínfimos.

Las dos líneas teóricas actuales de investigación mejor desarrolladas que buscan un esquema coherente para llegar a una teoría de gravedad cuántica son la teoría de las cuerdas y la gravedad cuántica de lazos (ciclos) (loop quantum gravity, también llamada "geometría cuántica»). Cómo ambas teorías usan la mecánica cuántica y la relatividad general, llegan a concluir, como escribe Carlo Rovelli (o. c. p. 168), que:

«[E]l espacio, siendo una entidad con propiedades dinámicas como el campo electromagnético, es en sí mismo también constituido por pequeños 
“quanta". Por otras palabras, según nuestro conocimiento actual del mundo natural, es bastante probable que el espacio no sea infinitamente divisible».

Es conveniente indicar aquí que, esta idea de granularidad finita del espacio no es sólo admitida por las teorías de cuerdas y de gravedad en loops, sino también por otras líneas de investigación en gravedad cuántica, como es el caso, por ejemplo, de las geometrías no-conmutativas y de la teoría de los conjuntos causales (causal set theory). Todas ellas admiten que el espacio no es infinitamente divisible.

Esta observación de la investigación en gravedad cuántica, la divisibilidad finita del espacio, indica que éste no es una cantidad continua. Se puede ilustrar eso utilizando de nuevo una analogía, como la de una tela tejida que, cuando se ve a cierta distancia parece una estructura continua, pero cuando se examina a una distancia más próxima revela ser una estructura con huecos. Así también una cierta cantidad de agua, la observamos y describimos a nivel macroscópico como poseyendo una estructura continua, pero a nivel microscópico la consideramos como un conjunto discreto de átomos. Análogamente, en las teorías de gravitación cuántica el espacio es pensado como un conjunto de átomos de espacio, cada uno de ellos conteniendo un volumen finito. En suma, el espacio no será infinitamente divisible y, en consecuencia, no existirá lo infinitamente pequeño en física.

Hasta aquí hemos analizado el problema de lo infinitamente pequeño en física, examinemos a continuación el problema del infinitamente grande a partir de la cosmología.

\section{LO INFINITAMENTE GRANDE Y LA COSMOLOGÍA}

Tradicionalmente, en cosmología el infinito aparece en forma de cuestiones relativas al espacio y al tiempo, cuestiones que son muy difíciles y que han llevado frecuentemente a paradojas. ¿Es el espacio infinitamente extenso? Si no los es, ¿qué es la frontera que lo limita? Pero, sí es infinito, ¿cómo puede ser objeto de investigación científica empírica? Cuestiones semejantes se aplican al tiempo, tales como, si el tiempo del Universo ha tenido un comienzo o si tiene una edad infinita. En el primer caso, ¿qué habría «antes» del Universo?

Recordemos que los antiguos griegos han especulado que el espacio tendría que ser infinito porque para ellos la noción de una extremidad o frontera para el espacio sería una noción inconsistente. De hecho, Arquitas de Tarento (430 a.C. - 360 a. C., matemático y astrónomo, pitagórico y contemporáneo de Platón) argumentaba de este modo: cuando mi mano llega al extremo del espacio, si nada hay en el extremo para bloquearla, el espacio continua; pero, si hay una "pared» para bloquearla, esa pared estará situada más-allá de la frontera, luego, el espacio continuaría ahí mismo en esa frontera. Por lo tanto, el espacio sería infinito e ilimitado.

Todavía, la moderna cosmología científica, que deriva de la TGR, coloca la posibilidad del Universo finito, pero ilimitado, con una forma de una tri-esfera 
(una superficie tridimensional doblada en una hiperesfera), solucionándose, así, la paradoja de Arquitas. De hecho, al darse un paso más allá de la frontera del Universo no se la sobrepasaría, sino que se volvería hacia atrás, permaneciendo dentro de él, justo como dar un paso más sobre la superficie esférica de la tierra permite permanecer sobre ella y regresar al punto de partida, describiendo un círculo máximo. Este es hoy un argumento para creer que el Universo puede ser finito pero ilimitado.

La cosmología científica es inaugurada con Einstein que, en su artículo de 1917, aplicaba sus ecuaciones de campo de la TGR a la totalidad del Universo, incluyendo enteramente ad hoc un nuevo término a sus ecuaciones, llamado constante cosmológica (una especie de fuerza repulsiva anti-gravitatoria). Este procedimiento le garantizaba que el Universo permanecía estático, con el fin de ajustarse a las ideas de ese tiempo, que no contemplaban un Cosmos en expansión.

\subsection{El modelo cosmológico estándar}

El Modelo Cosmológico Estándar del Big Bang (o de la gran explosión) es el modelo actual, basado en la cosmología relativista, que trata de comprender y de proporcionar una representación científica global del Universo, es decir, su geometría y estructura a gran escala y su dinámica evolutiva, desde sus primeros instantes. Es un modelo del Universo en expansión, a partir de una singularidad espacio-temporal, el Big Bang, situada en un pasado finito, a 13,8 x $10^{9}$ años. La expansión del Universo es descrita mediante modelos matemáticos, clásicos (por ejemplo, los modelos de Friedmann-Lemaître-Robertson-Walker - FLRW $)^{14}$, que son soluciones particulares de las ecuaciones de Einstein para la gravitación.

Las bases teóricas de este modelo son la TGR, el principio cosmológico —el cual afirma que, a gran escala, el Universo es homogéneo e isotrópico- y la hipótesis de que el Universo se comporta como un fluido en expansión, lo que implicaría la existencia de un tiempo inicial, $\mathrm{t}=0$, situado en un pasado finito, para el cual toda la masa cósmica estaría confinada en una región de radio muy pequeño, tendiente a cero, lo que no es físicamente aceptable.

El modelo cosmológico estándar es compatible con tres apoyos empíricos bien establecidos. El primero es la ley de Hubble, de 1929, para la recesión de

14 En 1922 el ruso Alexandre Friedmann aporta una solución para las ecuaciones de la TGR donde el Universo se expande y en 1924 presenta tres modelos de Universo con curvatura positiva (geometría esférica, Universo cerrado, espacial y temporalmente finito, pero ilimitado), cero (geometría euclideana, Universo plano, infinito temporal y espacialmente) y negativa (geometría hiperbólica, Universo abierto, infinito temporal y espacialmente). En 1927, Georges Lemaître, sacerdote católico belga, sin conocer los trabajos de Friedmann, consigue una solución similar, que describe un Universo de masa constante en expansión. En 1931 Lemaître llegó a la conclusión que el Universo podría tener un inicio temporal y haber nacido de una gran explosión de un "átomo primitivo», donde se encontraba condensada toda su masa. 
las galaxias (cuya luz se desplaza al rojo). El segundo es la radiación del fondo cósmico de microondas, descubierta por A. Penzias y R. Wilson en 1965. Esta radiación fue emitida cuando el Universo estaba lo suficientemente frío como para que se pudieran desacoplar materia y radiación y formar átomos neutros (unos 380.000 años después del BB). En 1992, con las mediciones del satélite COBE (Cosmic Background Explorer), se ha confirmado con mucha mayor precisión que esa radiación presenta un espectro perfecto de un cuerpo negro (que es un absorbedor y emisor perfecto de radiación) a una temperatura de 2,7 K, consiguiendo también detectar ínfimas anisotropías de temperatura, de una parte, en 100.000, que explican la formación de las galaxias. Los datos de COBE constituyen la piedra Rosetta cósmica que revela parámetros cruciales del Universo: su edad, densidad, geometría y composición química global. En 2003, las mediciones más precisas del satélite WMAP (Wilkinson Microwave Anisotropy Probe) han confirmado la existencia de esos datos, apuntado para una geometría plana ${ }^{15}$. El tercer apoyo empírico es la abundancia relativa de los elementos ligeros primigenios (isótopos de Hidrogeno, Helio y Litio) formados en las reacciones nucleares en la época de la nucleosíntesis.

La Agencia Espacial Europea (ESA) intenta actualmente construir el telescopio Euclides, cuyo lanzamiento espacial está previsto para 2020 y que podrá mapear galaxias hasta una distancia de 10.000 millones de años-luz. Al medir la influencia de la energía oscura sobre la distribución y la forma de las galaxias, se intentará verificar cómo la intensidad de esa energía ha variado a lo largo del tiempo.

\subsection{Otros elementos asociados al modelo cosmológico estándar}

Además de los anteriores pilares básicos del Modelo Cosmológico Estándar, se incorporan hoy otras características a fin de explicar la evolución y estructura actual del Universo. La primera es la inflación, una expansión enormemente acelerada, propuesta por Alan Guth, en 1981, y que explica la homogeneidad y la forma plana actuales del Universo.

En la década de 1970 la idea sencilla de una gran explosión inicial y posterior expansión del modelo de Big Bang no explicaba algunos hechos observacionales, tales como el problema del horizonte (debido a la finitud de la velocidad de la luz y del tiempo del Universo, hay un infinito número de regiones en direcciones opuestas del Universo que no han podido nunca comunicarse entre ellas, por lo que es difícil explicar la isotropía del Universo. Otro era el problema del Universo plano (relacionado con la medición de la densidad de materia del mismo).

15 Aunque los parámetros de las observaciones locales apunten para una geometría plana, no obstante, de ellos, no se puede necesariamente inferir esa geometría para las características globales del Universo. Esto se puede comprender mediante la siguiente analogía: una persona que analiza con instrumentos adecuados la topología de un terreno nos dirá que localmente (fuera de posibles accidentes del terreno) la superficie es plana, pero a nivel global (la Tierra) sabemos que ella es esférica. 
Para explicar estos problemas, Guth ha propuesto, con base en fluctuaciones cuánticas de la energía del vacío, que el Universo primitivo experimentó una brevísima fase temporal de expansión exponencial, llamada inflación ${ }^{16}$, en la que su tamaño crece muchísimo. Gracias a la inflación, toda la materia de actual Universo observable habría estado en contacto, solucionándose, así, el problema de la isotropía, y, a la vez, el problema de la planitud del Universo, ya que, durante la inflación, el crecimiento exponencial del espacio hace aplanar la curvatura del Universo, haciéndole plano.

La segunda característica es la «materia oscura fría» (CDM, Cold Dark Matter). Como se ha referido, aproximadamente un $27 \%$ del Universo debe estar formada por esta materia que actúa de forma gravitatoria, que no emite ni absorbe radiación electromagnética (es oscura) y fría (no debe moverse a velocidades relativistas). Esta materia es deducida por sus efectos gravitatorios en las galaxias y cúmulos de galaxias, particularmente sobre la rotación de las galaxias espirales. (Se piensa que la materia oscura pueda haber nacido de la sopa primitiva de los quarks, suponiendo que en esta se haya roto la simetría entre materia y antimateria, en favor de un ligero exceso de la materia.)

La tercera característica es la constante cosmológica (Lambda, $\Lambda$ ), que Einstein introdujo en sus ecuaciones de la TGR, originalmente para forzar que el Universo fuera estático, pero que hoy se piensa que pueda estar relacionada con la "energía oscura», recientemente descubierta, que es responsable por la expansión acelerada del Universo.

A partir de medidas efectuadas en fines del siglo pasado (1998) a las supernovas de galaxias distantes, se pudo concluir que el Universo sigue expandiéndose, a un ritmo acelerado, guiado por la energía oscura, una forma misteriosa y desconocida de energía que funciona como una anti-gravedad o repulsión cósmica, que puede ser asociada a la «constante cosmológica» y posiblemente a una misteriosa densidad de energía del espacio vacío. El término cosmológico de la ecuación de campo de la TGR ha sido asociado a esa energía oscura. Cálculos sencillos indican que la densidad de energía producida por la constante cosmológica sería 120 órdenes de magnitud mayor que aquella que parece existir realmente, lo que llevaría a un rápido despedazamiento del Universo y su consecuente inexistencia.

En 2003, las mediciones más precisas del satélite WMAP han confirmado la existencia de esos datos, deduciendo la cantidad de materia oscura un $23 \%$ de la energía oscura (un $72,5 \%$ ) y de la materia ordinaria (unos $4,5 \%$ ) y ayudado a establecer el paradigma del BB Inflacionario.

En 2009, el satélite Planck fue lanzado para hacer mediciones más precisas sobre el Universo primitivo y los orígenes de la formación de las estructuras cósmicas, habiéndose divulgado importantes resultados en 2013 y 2015. Esos

16 No existe hasta hoy un mecanismo físico suficientemente robusto teóricamente para justificar la inflación. Lo que tenemos son modelos demasiado sencillos desprovistos de fundamentos teóricos sólidos que, por ejemplo, no solucionan la cuestión de por qué el proceso inflacionario duró tan poco tiempo. 
resultados estiman un $68 \%$ de energía oscura, un $27 \%$ en de materia oscura y un 5\% de materia ordinaria, y validan los modelos de inflación, dando apoyo al modelo Lambda-CDM (es decir, el llamado "modelo concordante», con materia oscura fría, en el cual la energía oscura es compatible con la constante cosmológica, Lambda). Se puede decir que, actualmente, 95\% del Universo es aún desconocido.

\subsection{Breve descripción de la historia del universo}

describimos a continuación una brevísima historia del Universo, fundada en el modelo cosmológico estándar, añadiendo también algunos datos de la física de las partículas.

La cosmología actual sigue avanzando en la comprensión de la estructura, dinámica y génesis del Universo. Pero, el mayor desafío que plantea el avance del conocimiento de la génesis del Universo exige considerar simultáneamente los dos pilares de la física actual (la TGR y la MC) y reconciliarlos entre sí en una teoría cuántica de gravitación. Referimos ya anteriormente algunas líneas de investigación que buscan una teoría unificada de gravitación cuántica, particularmente las teorías de cuerdas y de la gravedad cuántica de lazos, sin haber, con todo, alcanzado hasta el presente, el amplio apoyo necesario de la comunidad de los físicos.

Sólo una teoría unificada podría aportar una comprensión de los primeros momentos del Universo, la llamada era de Planck, anteriores a los $10^{-43} \mathrm{~s}$ (que corresponde a distancias del orden de $10^{-33}$ centímetros y energías de $10^{19} \mathrm{GeV}$ ), cuando el espacio-tiempo empezó a tomar forma.

Aunque nuestros conceptos y leyes físicas se vean impotentes para describir el BB propiamente dicho y el origen último del Universo, ocurrido en un pasado finito situado a $13,8 \times 10^{9}$ años, el modelo estándar inflacionario actual describe su evolución desde los primeros instantes después de su «inicio» hasta el presente. Mirando hacia atrás en el tiempo, el Universo se presenta más denso, caliente, pequeño y sencillo, pero, tras el $\mathrm{BB}$, se va progresivamente expandiendo y enfriando.

Nuestro Universo pudo haberse originado por una fluctuación cuánticogravitatoria en la era de Planck, en la que las 4 interacciones fundamentales estaban unificadas. En seguida, es la era de la gran unificación $\left(10^{-43}-10^{-35}\right.$ s, después del BB), en la que el Universo se expande y enfría, separándose la gravedad de las otras 3 interacciones. Poco después, se da la era inflacionaria $\left(10^{-35}-10^{-32} \mathrm{~s}\right)$ de expansión exponencialmente acelerada. Avanzando más en el tiempo, el Universo era una sopa extremamente caliente, enormemente concentrada y casi uniforme de partículas elementales (quarks, leptones y los transmisores de las interacciones: fotones, bosones $\mathrm{W}$ y $\mathrm{Z}$ y gluones), que puede ser recreada en los grandes aceleradores de partículas. Esa sopa, que tuvo su origen en la fase de expansión inflacionaria, sufre un proceso de expansión lineal en el que se va enfriando, donde se van desarrollando en el curso del tiempo los niveles de estructura uno tras otro: los protones y 
neutrones, los núcleos atómicos, los átomos (esencialmente $\mathrm{H}$, un poco de $\mathrm{He}$ y vestigios de $\mathrm{Li}$ ), las galaxias, los cúmulos y los supercúmulos de galaxias, las estrellas, los planetas y la vida. Las galaxias y los cúmulos galácticos nacieron de fluctuaciones ínfimas de la densidad de la materia (una especie de semillas o irregularidades primordiales), bajo la acción de la gravedad, siendo que los gérmenes de tales fluctuaciones han dejado su huella en las irregularidades del ruido cosmológico de fondo.

\subsection{Los infinitos en cosmología}

Los infinitos que aparecen en cosmología relativista clásica (es decir, sin considerar la mecánica cuántica) están relacionados con el problema de la infinitud del espacio-tiempo, aunque, por razones de simplificación de los métodos matemáticos a utilizar, se considera separadamente en sus dos componentes: los infinitos en el espacio y en el tiempo. Básicamente hay dos tipos de infinitos en el espacio-tiempo: puntos o regiones «infinitamente distantes» (o sea, regiones infinitamente distantes del observador) y «singularidades» (o sea, regiones «infinitamente divergentes», que son regiones donde la estructura del espacio-tiempo se rompe, porque las magnitudes físicas tienden a infinito, como es el caso del Big Bang o de los agujeros negros, pero situadas a distancias finitas de nosotros en el espacio-tiempo). La estrategia de la cosmología es intentar conocer estas «regiones prohibidas» o «fronteras» (los infinitos de estos dos tipos) por aproximación sucesiva, a partir de las regiones regulares del espacio-tiempo.

¿Qué podemos decir sobre regiones infinitamente distantes, ya que tales regiones parecen inaccesibles? En realidad, para alcanzarlas necesitaríamos de un tiempo infinito o de enviarles mensajes con señales físicas propagándose a velocidades infinitas. Sin embargo, se puede conocer algo de algunas de estas regiones infinitamente distantes, pues las transformaciones conformes de la moderna geometría (que preservan los ángulos entre curvas y transforman en una proporción las grandezas congéneres) permiten operar un reescalonamiento en el espacio-tiempo, de tal modo que los puntos situados al infinito son llevados a un nivel finito, pudiéndose, así, investigar los «infinitos», mediante los patrones que ellos forman después de reescalonarlos. Pero, estas técnicas matemáticas tienen un precio a pagar, pues introducen distorsiones en las propiedades del espacio-tiempo, aunque con la excepción de que la estructura de propagación de la luz es mantenida, o sea, los fotones se propagan en geodésicas de tipo-luz. (o curvas nulas), que son invariantes bajo transformaciones conformes. A estas transformaciones se pueden juntar técnicas de compactificación y empleando diagramas de Penrose se pueden estudiar las estructuras de tipo-tiempo y de tipo-espacio y la estructura causal de varios espacios-tiempos. Todas estas técnicas matemáticas pretenden domesticar los infinitos.

Las singularidades infinitamente divergentes tienen la propiedad de que cuando nos aproximamos de ellas, la curvatura del espacio-tiempo y otras cantidades físicas tienden o divergen para infinito, lo que ocurre en los agujeros 
negros, como también en el modelo cosmológico del Big Bang, donde aparece una singularidad inicial en un pasado finito.

El concepto de agujero negro resulta de la misma TGR de Einstein, que predice que, cuando la materia se comprime suficientemente, su gravedad es tan intensa que delimita una región del espacio de la cual nada puede escapar, ni siquiera la luz, quedando todo (la materia y la luz) confinado en su interior ${ }^{17}$.

Los agujeros negros astrofísicos son los cadáveres de estrellas de masa elevada que se derrumbaron bajo su propia gravedad, o sea, por colapso gravitatorio estelar. Ellos no se observan directamente, pero pueden revelar su presencia, por ejemplo, cuando engullen la materia que pasa por sus proximidades. La materia así absorbida no se precipita al agujero negro en línea recta, sino en espiral, empezando por acumularse en un anillo aplastado, el llamado «disco de acreción». En el proceso se liberta una inmensa cantidad de energía bajo la forma de intensos rayos $\mathrm{X}$ y chorros gaseosos ${ }^{18}$. También se han descubierto concentraciones sorprendentes de masa-energía en el centro de galaxias activas (en evolución rápida) y en cuásares (núcleos de galaxias lejanas que son fuentes muy intensas de energía electromagnética, incluyendo radiofrecuencias e luz visible) que sugieren la presencia de agujeros negros gigantescos, que pueden alcanzar muchos millones de masas solares. Como referido anteriormente, la primera observación directa de agujeros negros ha sido realizada con la detección de ondas gravitacionales, el 14 de septiembre de 2015, por el observatorio norteamericano LIGO.

En los años setenta del siglo pasado, Stephen Hawking y Bernard Carr estudiaron un mecanismo para generar agujeros negros «primordiales», es decir, agujeros muy pequeños, microscópicos, en el Universo primitivo. Hawking descubrió que los agujeros negros no son completamente negros, pues emiten una radiación térmica, por lo que su masa tiende a disminuir, «adelgazando»,

17 En 1916, a los dos meses de la publicación de la TGR por Einstein, Karl Schwarzschild obtuvo una solución de las ecuaciones de Einstein que se han convertido en el prototipo mismo de agujero negro. El límite de esa región se llama horizonte (una frontera de causalidad) de sucesos del agujero negro. Los objetos pueden atravesarlo para caer dentro del agujero, pero ninguno lo atravesará de nuevo para salir de allá. El horizonte de un agujero negro divide la estructura del espacio-tiempo en dos partes: en el exterior y en el interior. Esta última se puede analizar estudiando la forma en que se propaga la luz. En los casos más simples, el tamaño del agujero es proporcional a su masa. Así, si se comprimiera el Sol hasta un radio de $3 \mathrm{~km}$ (el radio de Schwarzschild), unas cuatro millonésimas de su actual tamaño, se convertiría en un agujero negro (con una densidad del orden de $10^{19} \mathrm{~kg} / \mathrm{m}^{3}$ ). Para obtener el mismo efecto para la Tierra se necesitaría comprimirla hasta un radio de nueve milímetros, o sea, una milmillonésima de su tamaño actual. Así, cuanto más pequeño sea el agujero, más alto será el grado de compresión que se requerirá para para crearlo.

18 Eso se ha detectado en la observación de sistemas estelares binarios, es decir, de dos estrellas que giran una alrededor de la otra. Midiendo los parámetros dinámicos del sistema se puede conocer la masa del objeto emisor de rayos X y verificar se tal masa, siendo superior a la masa máxima compatible con una estrella de neutrones, indica la existencia de un agujero negro. 
y, de ahí, su inestabilidad que, con el tiempo, tendrá a evaporarse ${ }^{19}$. El estudio de agujeros negros primordiales, que puede que ni siquiera existan, llevó a difíciles paradojas al reconciliar la TGR con la $\mathrm{MC}^{20}$.

\section{4. ¿Infinitos universos?}

En las últimas décadas, para tratar el problema teórico del inicio del Universo, han surgido diversos modelos de Multiversos, basados en escenarios caóticos de inflación cosmológica y en la teoría de cuerdas. El físico ruso Andrei Linde ha propuesto, en 1986, un modelo de «inflación caótica» o «inflación eterna», partiendo de la idea de que las ecuaciones que describen la inflación sugieren que, si la inflación ha ocurrido una vez, debería ocurrir una y otra vez, con un número infinito de regiones inflacionarias originadas a lo largo del tiempo. Según este modelo, se podría pensar que las fluctuaciones cuánticas que originaran la inflación han tenido lugar en un espacio-tiempo preexistente y, además, procesos semejantes se podrían dar en nuestro propio Universo. De este modo, nuevos universos surgirían del nuestro, de la misma forma que éste habría aparecido de otro. Tendríamos, entonces, un proceso eterno, sin principio ni final, que iría produciendo, con diferentes probabilidades, universos independientes de diferentes tipos, con parámetros físicos distintos, incluyendo el número de dimensiones espaciales y los tipos de partículas estables. En otras palabras, el Multiverso estaría compuesto de burbujas individuales separadas e independientes de universos espacialmente infinitos en su interior, en número muy elevado o infinito, evolucionando en simultáneo, siendo que en algunos de ellos se darían, por azar, las condiciones físicas para la ocurrencia de la vida inteligente, como en el nuestro. En resumen, ¡tendríamos un número infinito de universos, y todos ellos serían físicamente realizables!

La idea de Multiverso parece dar respuestas a dos preguntas que nos podemos hacer: qué había antes de la gran explosión y por qué las leyes de

19 En 1974, estudiando el vacío cuántico (en el que nacen y desaparecen sin cesar pares de partículas y antipartículas virtuales) en el espacio-tiempo de un agujero negro, Hawking descubrió que los agujeros negros no son completamente negros, pues emiten una radiación térmica, con una temperatura inversamente proporcional a su masa. Como la emisión se lleva energía, la masa del agujero tiende a disminuir, «adelgazando», y, de ahí, su inestabilidad que, con el tiempo, tendrá a evaporarse, tiempo ese que es proporcional al cubo de su masa inicial. Por ejemplo, el tiempo de vida de un agujero de masa solar es de $10^{64}$ años, una inmensidad inobservable; el tiempo de vida de uno de $10^{12}$ kilogramos (tendría el tamaño de un protón) es de $10^{10}$ años, o sea, aproximadamente la edad del Universo.

20 La TGR afirma que la información sobre lo que cae en un agujero negro se pierde para siempre. Pero ¿qué sucede con la información que contiene en su interior cuando el agujero se evapora? ¿La información desaparece totalmente (como pretendía Hawking) en contra de la conservación de energía? ¿Los agujeros dejan remanentes o una especie de cicatriz en la estructura del espacio-tiempo? ¿Falla el principio de localidad? (según el cual la influencia entre acontecimientos espacialmente separados no puede ocurrir a velocidades superiores a la de la luz en el vacío). Los teóricos siguen sin encontrar respuesta para estas dificilísimas cuestiones. 
la física son como son (que es la célebre pregunta que Einstein hacia sobre si Dios podía elegir las leyes de la Naturaleza). La primera pregunta parece ya no tener sentido en el modelo de Multiverso, puesto que habrían existido infinitas grandes explosiones, cada una desencadenada por su propia inflación. No obstante, el problema ahora se complica mucho más, pues no se explica por qué hay infinitos universos (tal exigiría una explicación en cadena causal ad infinitum...). Además, no se explicaría, últimamente, por qué existe algo en lugar de nada, cuestión esta que nos obligaría a entrar en el ámbito de la metafísica. De igual modo, la segunda pregunta también parece carecer de sentido, pues la existencia de infinitos universos implicaría igualmente la existencia de todas las combinaciones de las leyes de la física, por lo que no habría ninguna razón especial para explicar las leyes particulares de nuestro Universo.

Hay todavía un problema mayor que enfrentan los modelos del Multiverso. Si los universos están realmente desconectados, no hay esperanza de testar experimentalmente su existencia. Comprendemos, pues, a este nivel, que se cruzan cuestiones fronterizas de física y de metafísica, que pueden derivar fácilmente para un terreno altamente especulativo.

La defensa de la idea del Multiverso encierra, algunas veces, el objetivo de neutralizar interpretaciones teleológicas del Universo, formuladas por el Principio Antrópico (hay varias versiones), el cual afirma que el Universo tiene, desde su inicio, unas características (sus leyes, parámetros y constantes universales, densidad, relación entre las fuerzas fundamentales, etc.), gracias a las cuales es posible la aparición de la vida inteligente (Brandon Carter, Martin Rees, J. Barrow and F. Tipler). Cuando nos preguntamos por la razón de que sea así, aparecen dos posibilidades: o el Universo tiene esas características «por casualidad» o ha sido diseñado con un ajuste fino (fine-tuning) por un Creador para nuestra existencia (la tesis del ajuste fino o del diseño inteligente). Aceptar la tesis de la casualidad no tiene sentido en el caso de un Universo, pues el azar es correlativo a la probabilidad de diversos resultados en muchos casos similares. Por eso, los proponentes de esta tesis acuden, consecuentemente, a la hipótesis de infinitos universos. No obstante, no explican por qué existe una infinitud real de objetos materiales, para la cual no se ve una justificación aceptable. Queda pues, la solución de que el Universo esté desde el comienzo finamente ajustado para la vida inteligente.

\section{CONCLUSIÓN}

En física, el problema de lo infinito aparece vinculado al problema de lo continuo de las representaciones matemáticas usadas y, por lo tanto, a la posibilidad, de la infinita divisibilidad (la división hasta lo infinitamente pequeño) y de la infinita extensión (la adición hasta lo infinitamente grande) de la geometría del espacio-tiempo y de los conceptos y magnitudes físicas mensurables. En otras palabras, el lenguaje matemático utilizado por la física, plantea, pues, la posibilidad de lo infinitamente grande y de lo infinitamente pequeño. 
La TGR, que es una teoría clásica, considera la geometría del espacio-tiempo y la interacción entre observador y observado en términos continuos, mientras que la MC, manteniendo aún la geometría espaciotemporal continua, admite que las cantidades mensurables características de los objetos cuánticos son discretas. De aquí resultan las dificultades actuales en unificar las dos teorías en una Teoría Cuántica de Gravitación satisfactoria, que pueda describir de modo totalmente coherente las cuatro interacciones conocidas para los fenómenos físicos.

El modelo estándar de las partículas considera que las partículas elementales son «infinitamente» pequeñas, es decir, singularidades espaciales, que no tienen estructura interna, ni dimensión mensurable (pues, no hay todavía experimentos que apunten para ello). Este modelo usa teorías locales de campos cuánticos, donde surgen cálculos con resultados infinitos para determinadas cantidades físicas. Para eliminar de los cálculos eses resultados infinitos, se utilizan métodos de renormalización. Los intentos de conjugar la física de partículas con la TGR, para distancias en la escala de Planck, se enfrentan con nuevos infinitos que surgen en los cálculos. Para eliminarlos, las teorías de cuerdas postulan unos objetos extensos con estructura, como los objetos más fundamentales de la teoría, pero, siguen encontrando singularidades.

En la cosmología relativista clásica (que no considera la MC) hay dos géneros de infinitos en el espacio-tiempo: las regiones «infinitamente distantes» y las «singularidades» (los agujeros negros y el inicio del Universo), donde la estructura suave y continua del espacio-tiempo se rompe, divergiendo los cálculos del infinito. Para ambos los casos se desarrollan métodos matemáticos de renormalización (tales como series convergentes hacia un límite, transformaciones conformes y otros más sofisticados) con la finalidad de domesticar los infinitos y, así, obtener una teoría o modelo más aceptable, que pueda ser corroborada por algún tipo de evidencia empírica.

En el modelo estándar cosmológico del BB, avalado por las observaciones empíricas (que validan también la inflación inicial y dan apoyo al modelo Lambda-CDM) y por la TGR (que usa la métrica FLRW, con tres tipos de modelos), la curvatura del espacio-tiempo depende de la densidad promedio de la materia-energía, siendo la densidad crítica $^{21}$ aquella para la cual el Universo es plano, es decir, con geometría euclidiana, espacialmente infinito y temporalmente infinito (tiene comienzo, pero no fin).

En el escenario de que la densidad del Universo sea inferior a la densidad crítica (Universo abierto, con geometría hiperbólica) o tenga exactamente esta densidad (Universo plano), seguirá expandiéndose sin límites de tiempo, enfriándose y, eventualmente, disgregándose (es el llamado Gran Desgarramiento o Big Rip). Tenemos, pues, infinitud espacial e infinitud temporal. La gravitación

21 Esta densidad es determinada por observación, juntamente con otros parámetros cosmológicos, como la constante de Hubble y la constante cosmológica. Notemos que no podemos observar el Universo globalmente como un todo, sino sólo un área limitada, local, determinado por la finitud de la velocidad de la luz. 
no consigue anular la expansión. Los modelos abierto y plano para el Universo consideran que este contiene una cantidad infinita de materia.

En el escenario inverso, en el que la densidad del Universo supere a la densidad crítica (Universo cerrado, con geometría esférica riemanniana) el Universo acabará por contraerse (pues la gravitación invertirá la marcha de la expansión) hasta concentrarse en una Gran Implosión (Big Crunch). El Universo será, pues, espacial y temporalmente finito, pero ilimitado. Este modelo cerrado de Universo admite una cantidad finita para la materia del Universo.

En varias líneas teóricas de investigación actual de gravedad cuántica (teoría de las cuerdas, gravedad cuántica de loops, geometrías no-conmutativas y de la teoría de los conjuntos causales) se considera con bastante probabilidad que el espacio, para dimensiones microscópicas, debido a sus propiedades dinámicas, no sea infinitamente divisible, sino que presenta una estructura descontinua o granularidad finita, constituida por «átomos» espaciales.

Por otra parte, los modelos de Multiversos, basados en argumentos probabilísticos y bastante más especulativos, suponen tiempos e espacios infinitos para el Universo, así como una colección infinita (posiblemente incontable) de Universos.

Como seres humanos finitos que somos, limitados por nuestros modelos científicos de cada tiempo ${ }^{22}$, siempre perfectibles, y por la finitud de nuestras observaciones empíricas - sea por las limitaciones de nuestras mediciones, sea por nuestra limitación fundamental del nuestro horizonte de visibilidad-, parece que no podemos probar nunca, en física, que el Universo es físicamente infinito. Afirmaciones fuertes sobre la infinitud del espacio, basadas en la aparente geometría plana, resultan un poco ingenuas.

El debate sobre si el Universo físico es finito o infinito ha estado siempre presente en la historia humana, desde los albores del pensamiento racional (referimos al comienzo los casos de Arquitas de Tarento y de Aristóteles). El infinito es ciertamente una de las mayores cuestiones con que el ser humano se enfrenta. En 1925, David Hilbert afirmaba que «el infinito siempre ha incendiado las emociones del ser humano (...) y estimulado y fertilizado la razón como pocas otras ideas lo han hecho» ${ }^{23}$.

El ser humano, siendo finito, tiene la idea de lo infinito. Más aún, tiene el deseo y la aspiración hacia el infinito, que no le dejan confinarse dentro de

22 Carlo Rovelli nos alerta para el peligro que existe en especular sobre el infinito a partir de nuestras teorías físicas, pues, según él, nada nos puede garantizar que «realmente existan Universos múltiplos, cuerdas, loops, y otros conceptos semejantes, y que, por ello, no deberíamos hacer deducciones demasiado fuertes basadas en la realidad de eses objetos». Rovelli, Carlo, «Some Considerations on Infinity in Physics», en: Heller, M. \& Woodin, H. (eds.), Infinity: New Research Frontiers, Cambridge University Press, Cambridge, 2011, p. 173.

23 Hilbert, David, «On the infinite», en: van Heijenoort, J. (ed.), From Frege to Gödel: A Source Book in Mathematical Logic, 1879-1931, Harvard University Press, Cambridge, MA, 1967, p. 371. 
ninguna medida finita. Por eso, el ser humano sigue recorriendo el asombroso viaje del conocimiento, elaborando modelos y teorías siempre más sofisticadas, descortinando posibilidades infinitamente insospechables, para superar sus límites finitos. El ser humano está, pues, estructurado y dirigido hacia el infinito. Él es capax infiniti, es capaz de lo infinito o, para decirlo en la bella formulación de San Agustín, es capax Dei, capaz de Dios. Y, por esto, el corazón del ser humano «vive inquieto, mientras no repose en Dios [lo infinito]» ${ }^{24}$.

\section{BibliografíA}

Aguirre, A. (2011). «Cosmological Intimations of Infinity», en: Heller, M. \& Woodin, H. (eds.), Infinity: New Research Frontiers. Cambridge: Cambridge University Press, pp. 176-192.

Arana, J. (2012). «Preguntas cosmológicas de los filósofos», en: Arana, Juan et. al. (ed.), La Cosmología en el Siglo XXI: entre la física y la filosofía. Tarragona: Publicaciones URV, pp. 143-159.

Aristóteles, Física, Consejo Superior de Investigaciones Científicas (CSIC), Madrid, 1996. Texto revisado y traducido por José Luis Calvo Martínez.

Balsas, Á. (2015). «O Infinito e a Busca de Fundamentos do Finito», en: Lind, Pedro (ed.), Cadernos de Mateus DOC VIII 8, Infinito / Infinity, Instituto Internacional Casa de Mateus (IICM), Mateus, Vila Real, pp. 203-239.

Bersanelli, M. (2011). «Infinity and the Nostalgia of the Stars», en: Heller, M. \& Woodin, H. (eds.), Infinity: New Research Frontiers. Cambridge: Cambridge University Press, pp. 193-217.

Börner, G. (2001). «Lo infinitamente grande», en: Investigación y Ciencia, Temas 23, Ideas del Infinito, $1^{\text {er }}$ trimestre, pp. 85-91.

Carr, B. J. \& Giddings, S. B. (2011). «Agujeros negros cuánticos», en: Investigación y Ciencia, Temas 63, Universo Cuántico, $1^{\text {er }}$ trimestre, pp. 36-43.

Dobrescu, B. A. \& Lincoln, D. (2016). «Materia oscura compleja», en: Investigación y Ciencia, Temas 84, Universo Oscuro, $2^{\circ}$ trimestre, pp. 14-21.

Ellis, G. F. R. (2007). «Issues in the Philosophy of Cosmology», en: Butterfield, Jeremy \& Earman, John (eds.), Philosophy of Physics. Amsterdam: part B, North-Holland and Elsevier, pp. 1183-1285.

Ellis, G. F. R. (2011). «Does the Multiverse Really Exist?», en: Scientific American, 305 (2), pp. 38-43.

Ellis, G. F. R. (2014). "On the philosophy of cosmology», en: Studies in History and Philosophy of Modern Physics, 46, pp. 5-23.

Fernández Barbón, J. L. (2011). "Geometría no conmutativa y espaciotiempo cuántico», en: Investigación y Ciencia, Temas 63, Universo Cuántico, $1^{\text {er }}$ trimestre, pp. 44-53.

Fritzsch, H. (2001). "Lo infinitamente pequeño en física», en: Investigación y Ciencia, Temas 23, Ideas del Infinito, $1^{\text {er }}$ trimestre, pp. 77-84.

Heller, M. (2011). «Infinities in Cosmology», en: Heller, M. \& Woodin, H. (eds.), Infinity: New Research Frontiers. Cambridge: Cambridge University Press, pp. 218-229.

24 Cf. San Agustín, Confesiones, I,1. 
Hilbert, D. (1967). «On the infinite», en: van Heijenoort, J. (ed.), From Frege to Gödel: A Source Book in Mathematical Logic, 1879-1931, Harvard University Press, Cambridge, MA, 1967, pp. 367-392. Tradução inglesa de «Über das Unendliche», Mathematische Annalen 95, pp. 161-190.

Ibañez, L. E. (2011). «Unificación y dualidad en teoría de cuerdas»", en: Investigación y Ciencia, Temas 63, Universo Cuántico, $1^{\text {er }}$ trimestre, pp. 28-34.

López Corredoira, M. (2012). «Ideas alternativas sobre cosmología en la ciencia actual», en: Arana, Juan et. al. (ed.), La Cosmología en el Siglo XXI: entre la física y la filosofía. Tarragona: Publicaciones URV, pp. 99-116.

Polchinski, J. (2016). "Agujeros negros y muros de fuego», en: Investigación y Ciencia, Temas 86, Fronteras de la Física Cuántica, $4^{\circ}$ trimestre, pp. 72-78.

Quigg, C. (2011) «Revolución en la física de partículas», en: Investigación y Ciencia, Temas 63, Universo Cuántico, $1^{\text {er }}$ trimestre, pp. 20-27.

Riess, A. G. \& Livio, M. (2016). "El rompecabezas de la energía oscura», en: Investigación y Ciencia, Temas 84, Universo Oscuro, $2^{\circ}$ trimestre, pp. 64-71.

Rovelli, C. (2011). «Some Considerations on Infinity in Physics», en: Heller, M. \& Woodin, H. (eds.), Infinity: New Research Frontiers. Cambridge: Cambridge University Press, pp. 167-175.

Tavakol, R. \& Gironi, F. (2016). "The infinite turn and speculative explanations in Cosmology», en: arXiv: 1604.06773v1[physics.hist-ph], pp. 1-14.

Tegmark, M. (2006). «Universos paralelos», en: Investigación y Ciencia, Temas 43, Fronteras de la física, pp. 14-26.

Trujillo, I. \& Sanromà, M. (2012). "La medición del Universo», en: Arana, Juan et. al. (ed.), La Cosmología en el Siglo XXI: entre la física y la filosofía. Tarragona: Publicaciones URV, pp. 35-56.

Trujillo, I. \& Sanromà, M. (2012). «El lado oscuro del Universo», en: Arana, Juan et. al. (ed.), La Cosmología en el Siglo XXI: entre la física y la filosofía. Tarragona: Publicaciones URV, pp. 57-75.

Turner, M. S., «El origen del universo», en: Investigación y Ciencia, Temas 63, Universo Cuántico, pp. 62-69.

Videira, A. L. L. (2016) “"Um dos Maiores Se Não Mesmo o Maior dos Feitos na História do Pensamento Humano": A Teoria da Relatividade Geral», Monografia, Centro Brasileiro de Pesquisas Físicas, CBPF, v. 2, n. 1, pp. 1-16.

Videira, A. L. L. (2004). «O Mundo que nos pertence», Ciência \& Ambiente, v. 28, Janeiro / Junho, pp. 135-154.

Videira, A. L. L. (2005). «A(s) Relatividade(s) de Einstein», Ciência \& Ambiente, v. 30, Janeiro / Junho, pp. 63-82.

Faculdade de Filosofia e Ciências Sociais

Universidade Católica Portuguesa, Braga

ÁlVARo BALSAS

abalsas@ucp.braga.pt

[Artículo aprobado para publicación en enero de 2018] 